\title{
NUMERICAL SIMULATION OF BLOOD FLOW IN ANEURYSMS USING A TWO-PHASE MODEL
}

\section{Md Al Amin Sheikh ${ }^{1}$, Anis Suhaila Shuib ${ }^{2}$ Mohd Hardie Hidayat Mohyi $^{2}$, Julaiha Adnan ${ }^{3}$ and Ahmad Sobri Muda ${ }^{4}$}

\author{
${ }^{1}$ School of Integrative and Global Majors, University of Tsukuba, 1-1-1 Tennodai, \\ Tsukuba, 305-8577, Ibaraki, Japan
}

\begin{abstract}
2School of Computer Science and Engineering, Taylors University, No 1, Jalan Taylors, 47500 Subang Jaya, Selangor, Malaysia
\end{abstract}
${ }^{3}$ Industrial Innovation Centre in Biomedical, SIRIM Industrial Research, SIRIM Berhad, Lot 5285, Lebuhraya Sg. Besi-Puchong, Sg.Besi, 57000 Kuala Lumpur, Malaysia

${ }^{4}$ Radiology Department, Hospital Pengajar Universiti Putra Malaysia, Faculty of Medicine and Health Sciences, UPM 43400 Serdang, Selangor, Malaysia

Corresponding Author's Email: ${ }^{1}$ s2130538@u.tsukuba.ac.jp

Article History: Received April 14, 2021; Revised June 8, 2021; Accepted June 14, 2021

\begin{abstract}
Computational fluid dynamics is utilized to understand the hemodynamics of aneurysms. In this study, two-phase blood flow was numerically simulated by a discrete phase model using the Lagrangian approach, with the blood cells representing the particulate phase and the plasma representing the continuous phase. Three patient-specific aneurysm geometries, namely, two saccular aneurysms located in the bifurcation of the basilar artery and one fusiform aneurysm located at the bifurcation of the common carotid artery and of the external carotid artery, were investigated. Wall shear stress (WSS), oscillatory shear index (OSI), and relative residence time (RRT) were determined from the simulation. The two-phase blood flow simulation revealed a high WSS $(>3.0 \mathrm{~Pa})$, a high OSI $(>0.2)$, and a long RRT $(>8)$ in the medium- and giant-sized saccular aneurysms. By contrast, a low OSI, a low WSS, and a short RRT were observed at the aneurysm dome of the fusiform aneurysm. However, at the bifurcation point, a high WSS and a long RRT with low oscillation were observed.
\end{abstract}

KEYWORDS: Aneurysm; Two-phase blood; Wall shear stress; Oscillatory shear 
index; Relative residence time

\section{$1.0 \quad 1.0$ INTRODUCTION}

A cerebral aneurysm is characterized by a bulging along the wall of a blood vessel in the brain. Such a condition weakens the vessel wall; moreover, aneurysms in cerebral arteries demonstrate the risk of rupturing or bursting [1], which would cause bleeding in the brain, known as hemorrhagic stroke. Understanding the mechanism of brain aneurysm rupture has been critical in finding the treatment to avoid the possible onset of fatal events [2]. Globally, nearly 500,000 deaths each year are caused by cerebral aneurysms [3].

Gaining an understanding of the biomechanics of blood flow is essential. Hemodynamics play a vital role in the formation, growth, and rupture of cerebral aneurysms [4]. Hemodynamics refers to the hydrodynamics of flow in blood vessels. It relates to the forces at play in blood flow. Several hemodynamic parameters are widely used in rupture risk analysis, in monitoring aneurysm growth, and in identifying atherosclerosis lesions; moreover, these parameters are used as indicators when determining the location where aneurysm formation would peak [5] [6] [7] [8].

Over the last two decades, computational fluid dynamic (CFD) techniques have been widely used to investigate the initiation, growth, and rupture of cerebral aneurysms [9]. Rupture risk prediction has been extensively investigated using CFD simulation wherein blood has been analyzed using a single-phase flow model, which considers hemodynamic parameters, such as wall shear stress (WSS) and oscillatory shear index (OSI); relative residence time (RRT) is a parameter that has been less investigated [8] [10]. In single-phase blood flow models, blood is treated both as a Newtonian and a nonNewtonian fluid in order to understand the development, growth, and rupture of cerebral aneurysms [11] [12]. Blood has also been analyzed using a multiphase flow model, wherein red blood cells (RBCs), white blood cells (WBCs), and platelets are considered to be suspended in the plasma. Additionally, in one study, cell transport, interactions, and low WSS distribution in leukocytes were analyzed in four patient-specific aneurysm geometries [13].

In this study, blood was considered as a two-phase fluid, with plasma as the continuous phase and RBCs as the particulate phase. The continuous phase was solved using the Naiver-Stokes equation, and 
the particulate phase was solved using the discrete phase model (DPM). This research aims to determine the WSS, OSI, RRT, velocity streamlines, and surface velocities in aneurysms by using a two-phase blood flow model.

\subsection{METHODS}

\subsection{Equations and validation of the DPM}

The ANSYS-Fluent simulation package with DPM was utilized to solve the motion of plasma and RBCs using the Lagrangian approach, wherein blood trajectories were calculated. The continuous fluid was first solved according to the Naiver-Stokes equation, and the particulate phase in the plasma was solved using the DPM. The individual particles were tracked by calculating the fluid flow field. Each particle was analyzed computationally at certain time intervals during the fluid phase calculation. The trajectory equation was solved through stepwise interactions over discrete time steps. The integration of time yielded the velocity of the particle at each point along the trajectory.

In the DPM, the blood cells were considered rigid particles. The movement of blood cells is governed by particle motion. The equation for particle motion was fundamentally derived from Newton's laws of motion, where the resultant of all the forces $\Sigma F$ acting on particles' mass $m$ is proportional to the acceleration $a$ of the particles, and each action has a reaction that is equal in magnitude but acting in the opposite direction.

$$
\Sigma F=m a
$$

The motion of $a$ spherical particle in a fluid is expressed as follows:

$$
m_{p} \frac{\partial u_{p}}{\partial t}=m_{p}\left(1-\frac{\rho}{\rho_{n}}\right) g+F_{p g}+F_{D}+F_{L}+F_{v m}+F_{\text {Bas }}
$$

where $m_{p}$ is the mass of the particle, $u_{p}$ is the instantaneous velocity of the particle, $g$ is the body acceleration, $F_{D}$ is the drag force, $F_{L}$ is the lift force generated by the rotation of a particle and shear flow, $F_{p g}$ is the force that exists in the absence of a particle due to the acceleration of the fluid and due to the hydrostatic pressure gradient, $F_{v m}$ is the virtual mass force that accounts for the work required to change the momentum of the surrounding fluid as particles accelerate, and $F_{B a s}$ is the unsteady drag force or Basset force that accounts for the temporal development of the viscous region that affects the velocity of the particles. 
An equal balance between particles' mass acceleration and the action forces was used to determine the movement of each particle in the disbanded phase. In this research, we presumed the force as a drugrelated force, whereas all other forces were considered small and negligible [14]. The governing equation is as follows:

$$
m_{p} \frac{\partial u_{p}}{\partial t}=m_{p}+\left(1-\frac{\rho}{\rho_{n}}\right) g+F_{D}
$$

where $F_{D}$ is the per unit mass drag force and is derived as

$$
F_{D}=\frac{18 \mu}{\rho_{n} d_{n}^{2}} \frac{C_{d} R e_{p}}{24}\left(u-u_{p}\right)
$$

where $u$ is the fluid velocity, $\rho$ is the fluid density, $\rho_{p}$ is the particle density, $d_{p}$ is the particle density, $C_{d}$ is the drag coefficient, and $R e_{p}$ is the relative Reynolds number, which is expressed as

$$
R e_{p}=\frac{\rho a_{p\left|u-u_{p}\right|}}{\mu}
$$

The CFD software uses the empirical approach described by Morsi and Alexander [15] to determine the drag coefficient, which is defined as follows:

$$
C_{d}=\omega_{1}+\frac{\omega_{2}}{R e_{n}}+\frac{\omega_{3}}{R e_{n}^{2}}
$$

where $\omega_{1}, \omega_{2}$, and $\omega_{3}$ are the constants that are appropriate for the spherical particles at different Reynolds numbers. The particle trajectory equation is expressed as follows:

$$
\frac{\partial x_{i}}{\partial t}=u_{p . i}
$$

The particle time is $t$, and $x_{i}$ is the position coordinate, as described by Dhahbi et al. 2012 [14]. The interaction of the injected particle with the continuous phase was specified, and 10 continuous phase iterations per DPM iteration were defined per the software guidelines [16].

To validate the DPM, we performed CFD simulation by benchmarking with an experimental study [17], along with the CFD results for patient-specific cerebral aneurysms and arterial blockage [13] [18] [19]. Also, a numerical validation of the DPM was published elsewhere [21]. In the validation study, blood was divided into two major components: water and RBCs. Human RBCs, which had a density of $1130 \mathrm{kgm}^{-3}$ and a diameter of $7.5 \mu \mathrm{m}$, were considered as rigid particles that are suspended in water, which had a density and viscosity of $1000 \mathrm{kgm}^{-3}$ and $0.001 \mathrm{~Pa} \cdot \mathrm{s}$, respectively [20] . Two inlet 
velocities, $0.0757 \mathrm{~ms}^{-1}$ and $0.233 \mathrm{~ms}^{-1}$, were chosen; the fluid flow was considered Newtonian with a steady-state flow. As shown by theoretical and experimental studies, the reattachment point plays an important role in the numerical validation of the DPM. Figure 1 compares the reattachment points obtained in an experimental work, in two computational studies, and in the current computational investigation. The validated DPM model was used in this simulation.

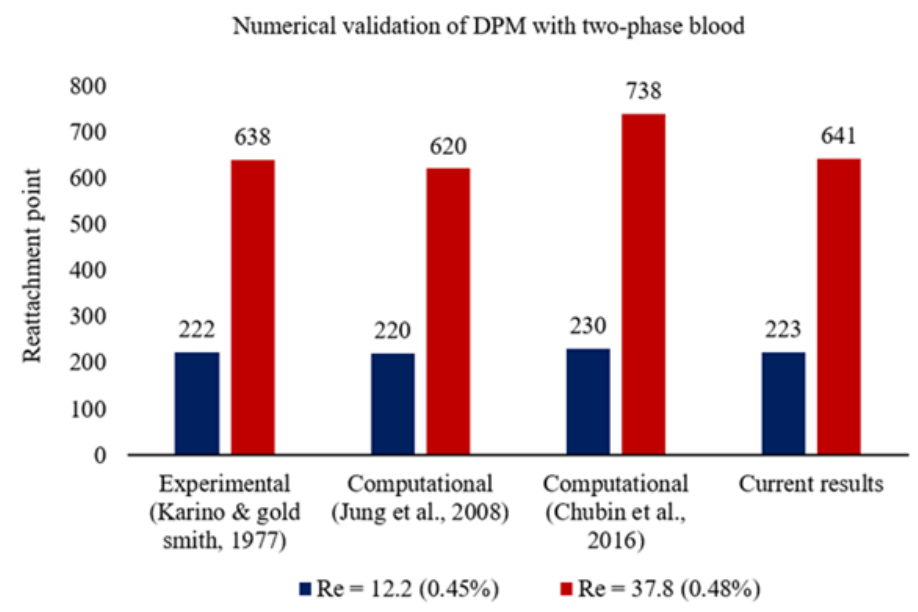

Figure 1: Numerical validation of the DPM, wherein the current results are compared with previous findings.

\subsection{Patient-specific aneurysm geometries}

Three patient-specific aneurysm geometries were selected to determine the rupture risk of cerebral aneurysms. These patient-specific aneurysm geometries were segmented and constructed from 3D rotational computed tomography angiography (CTA) images to generate 3D geometries using the medical research software Materialise Mimics 22.0 and 3-Matic 20.0. Data were obtained from the Radiology Department, Faculty of Medical and Health Sciences, Universiti Putra Malaysia. The segmented aneurysm geometries denoted as geometry 1 , geometry 2 , and geometry 3 , are shown in Figure $2 \mathrm{a}, 2 \mathrm{~b}$, and $2 \mathrm{c}$, respectively.

The aneurysm geometries 1 and 3 were saccular aneurysms located in the bifurcation of the basilar artery (BA) to the posterior communicating arteries. The aneurysm geometry 2 was a fusiform aneurysm located at the bifurcation of the common carotid artery (CCA) and external carotid artery. However, missing information in the CTA images in aneurysm case 3 , that is, the outlet 1 artery with aneurysm geometry, was reconstructed for further investigation. 


\subsection{CFD method}

The Ansys-Fluent 2019 R3 was used for the CFD analysis of the patientspecific aneurysm geometries. An unstructured tetrahedral mesh with a maximum element size of $0.15 \mathrm{~mm}$ with a four-layer prism mesh was applied after completing the mesh independence test. The meshed aneurysm geometry 2 is shown in Figure $2 \mathrm{~d}$ to $2 \mathrm{f}$. The continuous phase was the plasma, which had a density of $1020 \mathrm{kgm}^{-3}$ and a viscosity of $0.002 \mathrm{~Pa} \cdot \mathrm{s}$. The particulate phase had a mean diameter of $8.2 \mu \mathrm{m}$ and a density of $1100 \mathrm{kgm}^{-3}$ [17].
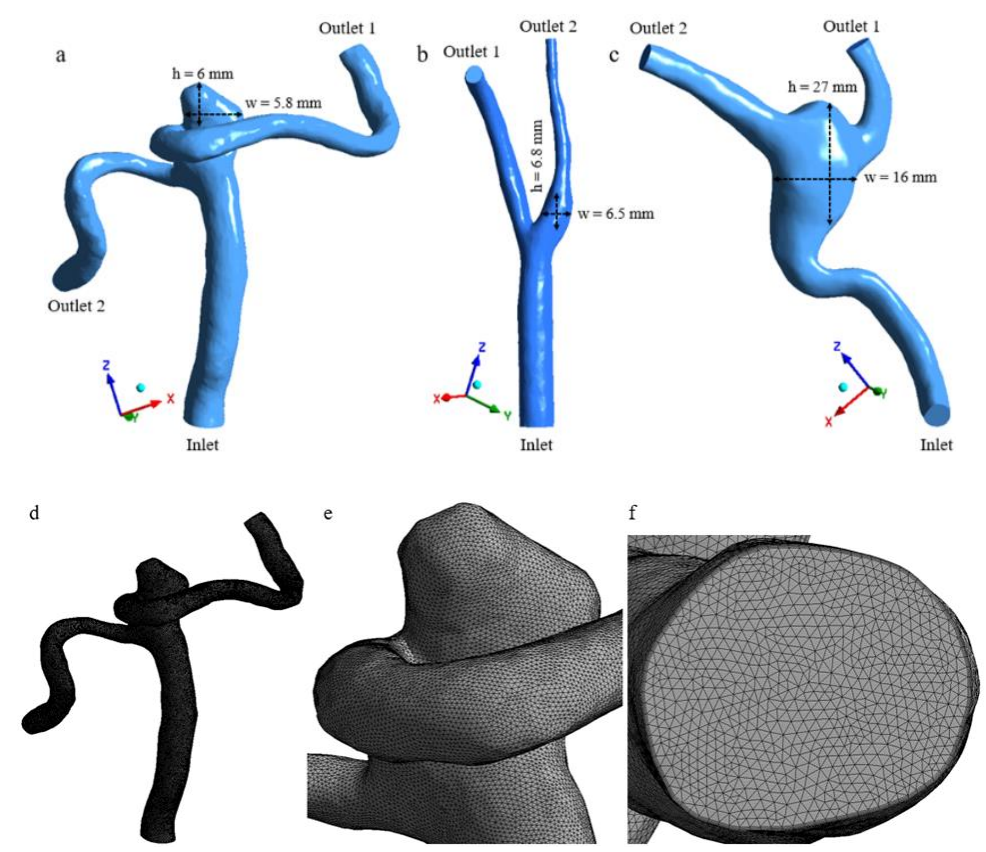

Figure 2: Patient-specific aneurysm geometries. (a) Geometry 1 (height $(\mathrm{h})=6 \mathrm{~mm}$; width $(\mathrm{w})=5.8 \mathrm{~mm})$; (b) geometry $2(\mathrm{~h}=6.8 \mathrm{~mm}$; $\mathrm{w}=6.5 \mathrm{~mm})$; and $(\mathrm{c})$ geometry $3(\mathrm{~h}=27 \mathrm{~mm} ; \mathrm{w}=16 \mathrm{~mm})$. Meshed aneurysm geometry 1: (d) full geometry of the unstructured tetrahedral mesh, (e) aneurysm body mesh, and (f) inlet meshing with a four-layer prism mesh.

The blood flow in the aneurysm was modelled to be incompressible. A pressure-based solver was selected for the simulation. The SemiImplicit Method for Pressure Linked Equation (SIMPLE) scheme was used for pressure-velocity coupling. A 1s pulsatile inlet velocity profile was defined and written as the user-defined function in the $c$ programming language [22]. Figure $3 a$ presents the inlet pulsatile 
velocity profile. Zero pressure was applied in the outlet boundary condition. Ten cardiac cycle simulations were performed for each aneurysm geometry with a time-step of $0.005 \mathrm{~s}$, as shown in Figure $3 \mathrm{~b}$. Each time-step convergence achieved a minimum residual of $10^{-4}$.
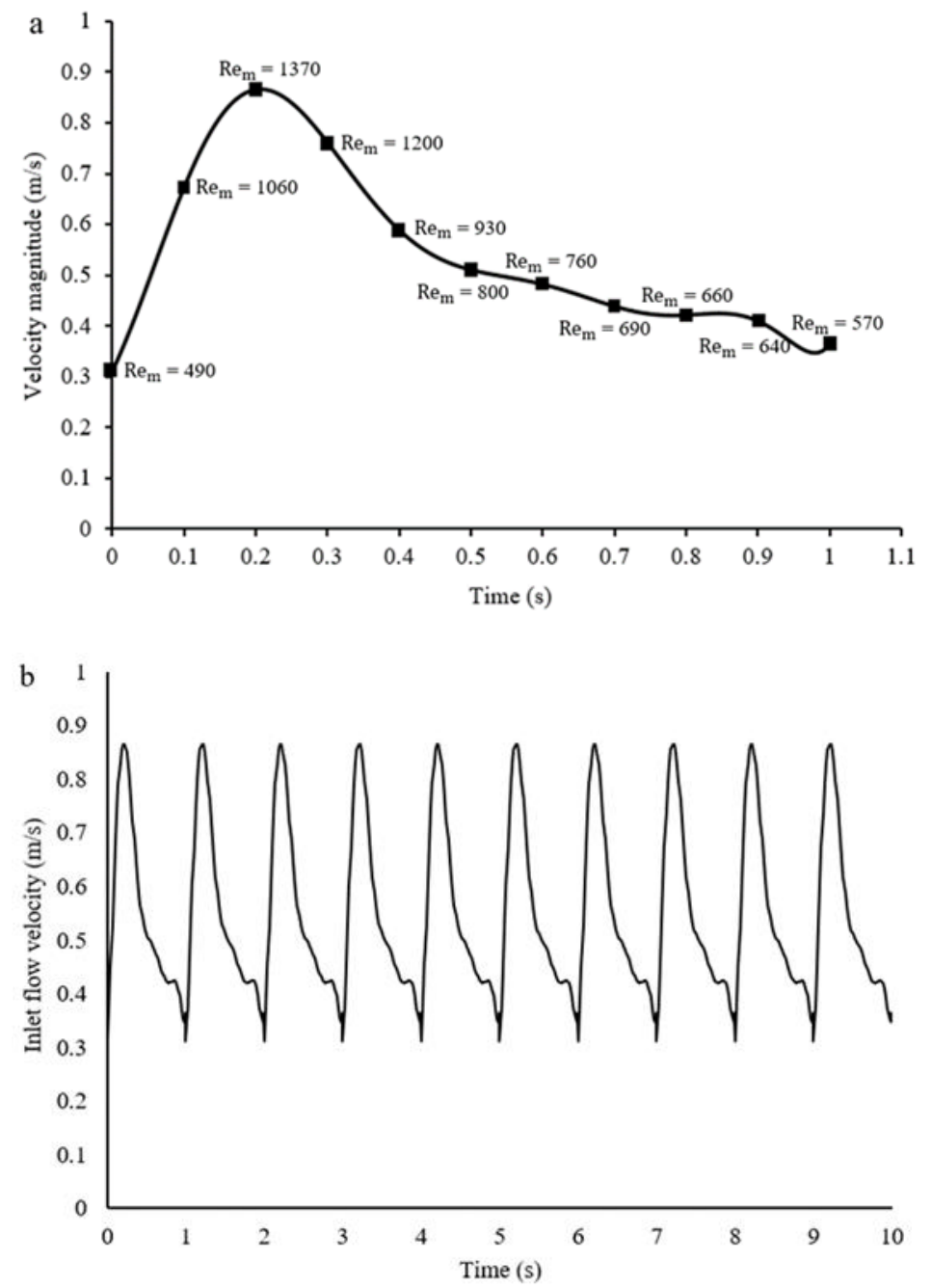

Figure 3: Inlet pulsatile velocity profile. (a) 1s inlet pulsatile velocity with mean Reynolds number. (b) Inlet flow velocity in 10 cardiac cycles.

The Reynolds number was based on the parent artery's mean inlet diameter and was calculated using the following equation: 


$$
R e_{m}=\frac{\rho D_{\text {mean }} V}{\mu}
$$

where $\rho$ is the density of the fluid, $D_{\text {mean }}$ is the mean diameter of the parent blood vessels inlet, $V$ is the velocity of the fluid, and $\mu$ is the fluid viscosity. The mean Reynolds numbers $\operatorname{Re}_{m}$ of the three aneurysm geometries ranged from 490 to 1370 . The data were based on the mean Reynolds number obtained in the simulation that involved 10 cardiac cycles.

The hemodynamic parameters obtained from the simulation were WSS (calculated as the ratio of the time-averaged WSS of the last cardiac cycle to the average WSS of the parent artery), OSI (a nondimensional parameter calculated based on the directional changes in WSS), and RRT (residence time of blood cells near the artery of the aneurysm wall). To analyze the velocity distribution inside the aneurysm, we retrieved the velocity streamline and surface velocity data for all aneurysm geometries.

\subsection{RESULTS}

The two-phase blood was solved with the DPM and completed in 10 cardiac cycles. After the simulation for 10 cardiac cycles, a total of 145,865 RBCs for aneurysm geometry 1, 160,245 RBCs for aneurysm geometry 2, and 185,715 RBCs aneurysm geometry 3 were tracked.

Large aneurysms possibly have a higher risk of rupture compared with the small ones [23]. According to Lee et al. 2015 [24], aneurysm sizes are classified based on the maximum dimension of aneurysm geometries. The size categories are as follows: small $(0 \mathrm{~mm}$ to $4.9 \mathrm{~mm})$, medium (5 $\mathrm{mm}$ to $9.9 \mathrm{~mm})$, large $(10 \mathrm{~mm}$ to $24 \mathrm{~mm})$, and giant $(>25$ $\mathrm{mm}$ ). On this basis, the aneurysm geometries 1 and 2 were classified as medium-sized aneurysms, whereas geometry 3 was classified as a giant aneurysm. The height and width of the aneurysm geometries are presented in Figure 3. WSS, OSI, RRT, velocity streamlines, and surface velocities for the last cardiac cycle for geometries 1,2, and 3 are plotted in Figures 4, 5, and 6, respectively.

WSS is an essential hemodynamic parameter and is categorized as either high or low. A low WSS is characterized by $<1 \mathrm{~Pa}$, whereas a high WSS is characterized by $>3 \mathrm{~Pa}$ [23]. In the two-phase blood flow simulation, the high WSS values were observed in aneurysm geometries 1 and 3, and a low WSS was observed in the aneurysm dome of geometry 2, as shown in Figures 4a, 6a, and 5a, respectively. A high WSS was observed at the bifurcation in aneurysm 2 . 


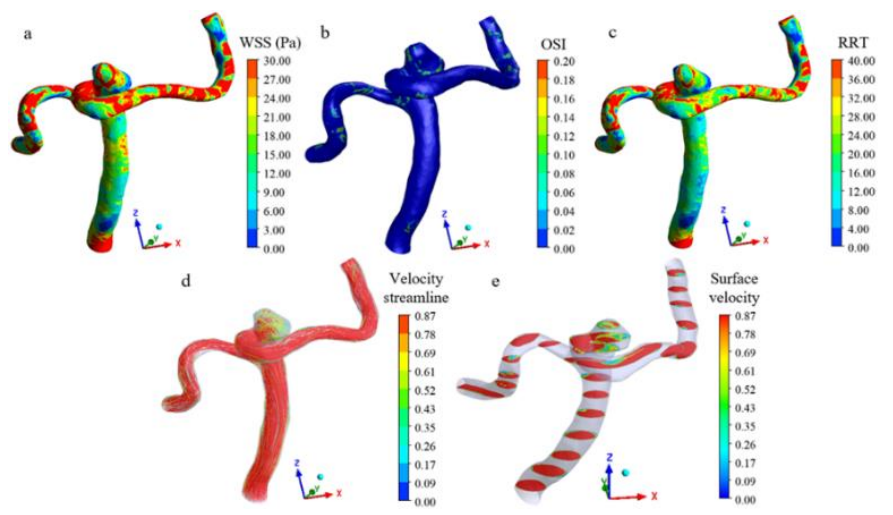

Figure 4: Geometry 1: Medium-sized saccular aneurysm in the BA. (a) WSS. (b) OSI. (c) RRT. (d) Velocity streamlines. (e) Surface velocities.

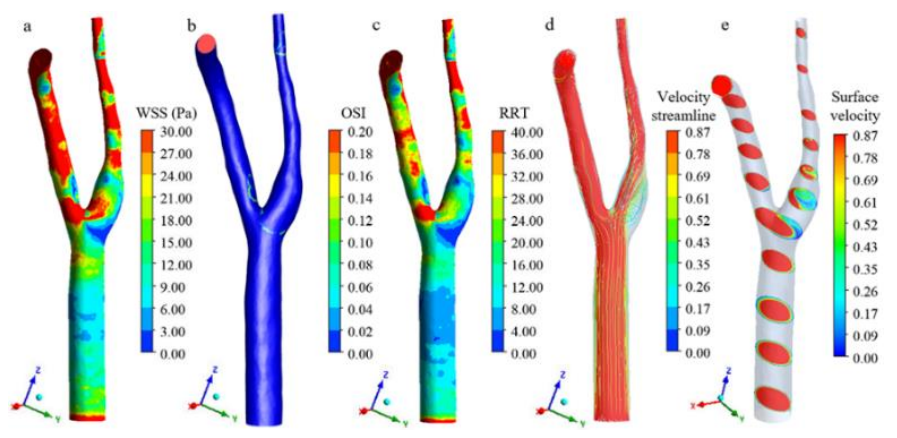

Figure 5: Geometry 2: Medium-sized fusiform aneurysm in the bifurcation of the CCA. (a) WSS. (b) OSI. (c) RRT. (d) Velocity streamlines. (e) Surface velocity. 


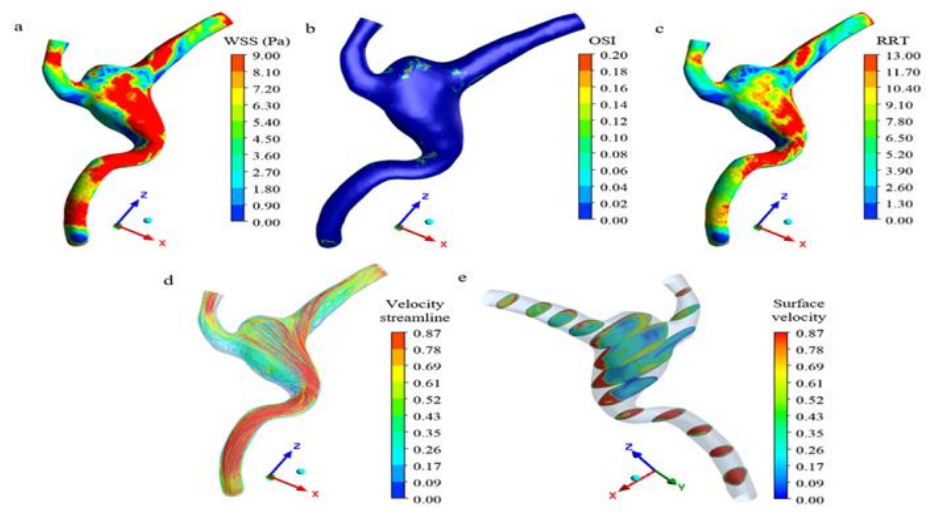

Figure 6: Geometry 3: Giant saccular aneurysm in the BA. (a) WSS. (b)

OSI. (c) RRT. (d) Velocity streamlines. (e) Surface velocity.

The OSI, which ranges from 0 to 0.2 , indicates the directional changes in the WSS. Zero indicates no oscillation, whereas 0.2 indicates high oscillation [24] [25]. High OSI values were observed in the aneurysm geometries 1 and 3, and a low OSI was observed in the aneurysm dome of geometry 2 , as shown in Figures $4 b, 6 b$, and $5 b$, respectively.

The RRT indicates the residence time of blood flow inside an aneurysm. A short RRT is indicated by values 0 to 7 , whereas values $>8$ indicate a long RRT [26]. A long RRT was observed in the aneurysm geometries 1 and 3, whereas a low RRT was observed in the aneurysm dome of geometry 2, as shown in Figures 4c, 6c, and 5c, respectively. Also, a long RRT was observed at the bifurcation of geometry 2.

Overall, a high WSS, a high OSI, and a long RRT were observed in the aneurysm dome of geometries 1 and 3. In geometry 2, a low WSS, a low OSI, and a short RRT were observed in the aneurysm dome, whereas a high WSS and a long RRT were observed at the vessel bifurcation point.

Velocity streamline and surface velocity were calculated to analyze the velocity distribution inside the aneurysms. To calculate the velocity distribution inside the aneurysm, we calculated the contour between 0 and $0.865 \mathrm{~ms}^{-1}$. The velocity streamline indicates the blood flow circulation inside the aneurysms. Figures $4 \mathrm{~d}$ and $4 \mathrm{e}$ show the changes in velocity streamline and surface velocity, respectively, for the aneurysm geometry 1 . As blood was recirculating, and variable high velocities were observed based on the velocity streamline and surface velocity changes in the aneurysm dome. For the aneurysm geometry 2 , the velocity streamline indicates the flow stagnation in aneurysm shown in Figure 5d. Changes in surface velocity were observed in the aneurysm, and the middle surface of the aneurysm demonstrated low- 
velocity values, as depicted in Figure 5e. As displayed in Figure 6d, flow disturbance and recirculation were observed in geometry 2 , which is a giant aneurysm in the BA. The high velocity in the aneurysm dome is shown in Figure 6e.

\subsection{DISCUSSION}

Hemodynamics is important in predicting the risk of aneurysm rupture in cerebral arteries when using a single-phase blood flow model. WSS, OSI, and RRT are the key hemodynamic parameters that are directly related to aneurysm rupture risk in cerebral arteries [28]. However, in a single-phase, blood flow simulation, variations in high and low WSS values were not comprehensively investigated as to whether they describe the properties of a Newtonian and a non-Newtonian fluid [25]. In this study, a two-phase blood flow simulation was performed using three patient-specific aneurysm geometries. The two-phase blood that consists of plasma and RBCs plays an essential role in predicting rupture risk, which is indicated by a high WSS, a high OSI, and a long RRT. Jung et al. 2008 [18] observed a high velocity with high WSS values in plasma containing RBCs, whereas a low WSS was observed in WBCs in the multiphase blood flow model of Chubin et al. 2016 [13]. A high WSS, a high OSI, and a long RRT may be associated with a high risk of aneurysm rupture in cerebral arteries.

WSS is an essential hemodynamic parameter in rupture risk prediction in brain vessels with an aneurysm. For the rupture risk analysis, WSS was calculated mainly using the normalized WSS and time-averaged WSS in cerebral arteries with aneurysms [5] [24]. The WSS contour value was normalized based on the actual WSS values, and the values obtained ranged from 0 to 1 or 2.5 [24] [29]. The timeaveraged WSS was calculated using the characteristics of an entire-time cycle and was measured in pascal, with normal values ranging from 1.5 to 10 based on the absolute time-averaged magnitude of each wall mesh point vector [5]. As for the WSS in brain vessels with aneurysms, the contour value of the WSS is influenced by various parameters, such as blood vessel geometry, meshing, and blood cell properties. Usually, the blood pressure is 10,000 Pa when the WSS of the wall of a healthy vessel is $0 \mathrm{~Pa}$ to $20 \mathrm{~Pa}$ [30]. Valen-Sendstad et al. 2011 [22] simulated the average pulsatile blood flow in a middle cerebral artery aneurysm. The WSS contour was $0 \mathrm{~Pa}$ to $48 \mathrm{~Pa}$ based on the average pulsatile blood. In the aneurysm wall, the normal WSS values were $0 \mathrm{~Pa}$ to $1.5 \mathrm{~Pa}$, and a WSS of $1.5 \mathrm{~Pa}$ to $2.0 \mathrm{~Pa}$ indicated a high risk of rupture. A value greater than 3.0 Pa is considered a high WSS [31].

Some studies have found that both high and low WSS were related to aneurysm growth and rupture [23]. However, the present study focuses on high WSS values in the aneurysm wall because the twophase blood plasma containing RBCs is associated with high WSS. 
High WSS in turn is associated with positive WSS gradient resulting from endothelial cell damage, matrix metalloproteinase production by mural cells, extracellular matrix degradation, thinning of media, and apoptosis of smooth muscle cells and fibroblasts, and such an association further increases the risk of aneurysm rupture [27].

OSI, a non-dimensional parameter whose value ranges from 0 to 0.5 , is used to calculate the directional changes in WSS in a cardiac cycle. In this study, OSI ranged from 0 to 0.2 , consistent with the findings of previous studies, where 0 represents a steady flow and 0.2 represents a high oscillation in aneurysm geometries [25]. In an aneurysm, OSI indicates several directional changes throughout a cardiac cycle. A low OSI may promote atherogenesis and inflammatory diseases and may lead to intimal wall thickening [27] [32] [26]. However, in several studies, a high OSI has been observed in ruptured aneurysms, and it has been an independent risk factor in aneurysm rupture risk prediction [27] [26] [33].

The RRT is the residence time of blood near the aneurysm wall. The RRT was based on a low WSS and a high OSI inside the aneurysm. Long-term circulation of blood flow near the aneurysm wall could lead to the rupture of an aneurysm. A study involving 30 aneurysm cases investigated the RRT, which is an essential indicator of atherosclerotic changes in cerebral aneurysms [34]. The occurrence of a low WSS with long RRT was investigated in atherosclerotic and non-atherosclerotic aneurysms, respectively; long RRT was observed in atherosclerotic aneurysms but not in non-atherosclerotic aneurysms [7]. However, knowledge on the association between a high WSS with a long RRT and the high risk of rupture remains limited. The long residence time of blood cells triggers the cellular interaction that promotes blood clotting and wall dilation. A high WSS with a long RRT is a key indicator that leads to a high risk of aneurysm rupture in cerebral arteries.

Table 1 presents the rupture risk assessment of the aneurysm geometries based on WSS, OSI, and RRT with mean Reynolds number. The simulation results for the last cardiac cycle are based on the inlet mean Reynolds number, which indicates the risk (i.e., high or low) of aneurysm rupture. The aneurysm geometries 1 and 3 demonstrate a high risk of rupture compared with the aneurysm geometry 2 . In geometry 2, a high WSS, a low OSI, and a long RRT were observed at the vessel bifurcation point. According to Soldozy et al. 2019 [27], an aneurysm has a low risk of rupture, but further growth of the aneurysm could be anticipated.

Table 1: Rupture risk analysis of the aneurysm geometries based on the changes in WSS, OSI, and RRT with mean Reynolds number. 


\begin{tabular}{|c|c|c|c|c|}
\hline $\begin{array}{l}\text { Aneurysm } \\
\text { geometries }\end{array}$ & WSS & OSI & RRT & Analysis \\
\hline $\begin{array}{l}\text { Aneurysm } \\
\text { geometry } 1\end{array}$ & $\begin{array}{l}\text { High WSS at } \\
R e_{m} \text { in } \\
\text { between } 1200 \\
\text { to } 1370\end{array}$ & $\begin{array}{c}\text { High OSI at } \\
R e_{m}=1060, \\
1200,760, \text { and } \\
660\end{array}$ & $\begin{array}{c}\text { Long RRT } R e_{m}: \\
1200<R e_{m}< \\
1370\end{array}$ & $\begin{array}{l}\text { High risk } \\
\text { of rupture }\end{array}$ \\
\hline $\begin{array}{c}\text { Aneurysm } \\
\text { geometry } 2 \\
\text { (aneurysm } \\
\text { dome) }\end{array}$ & Low WSS & Low OSI & Short RRT & $\begin{array}{l}\text { Low risk } \\
\text { of rupture }\end{array}$ \\
\hline $\begin{array}{c}\text { Aneurysm } \\
\text { geometry } 2 \\
\text { (vessel } \\
\text { bifurcation } \\
\text { point) }\end{array}$ & $\begin{array}{l}\text { High WSS at } \\
R e_{m} \text { in } \\
\text { between } 1200 \\
\text { to } 1370\end{array}$ & Low OSI & $\begin{array}{c}\text { Long RRT } R e_{m}: \\
1200<R e_{m}< \\
1370\end{array}$ & $\begin{array}{l}\text { Low risk } \\
\text { of rupture. } \\
\text { Aneurysm } \\
\text { growth } \\
\text { may occur. }\end{array}$ \\
\hline $\begin{array}{l}\text { Aneurysm } \\
\text { geometry } 3\end{array}$ & $\begin{array}{c}\text { High WSS at } \\
R e_{m} \text { in } \\
\text { between } 800 \text { to } \\
1370\end{array}$ & $\begin{array}{c}\text { High OSI at } \\
R e_{m}=1370, \\
1060,930,760, \\
\text { and } 690\end{array}$ & $\begin{array}{c}\text { Long RRT } R e_{m}: \\
1200<R e_{m}< \\
1370\end{array}$ & $\begin{array}{l}\text { High risk } \\
\text { of rupture }\end{array}$ \\
\hline
\end{tabular}

Previously, a high WSS, a high OSI, and a long RRT were reported as key indicators that are highly associated with a damaged artery wall and that may promote aneurysm rupture [27] [26] [31].

\subsection{LIMITATION}

This study has some limitations. The 3D rotational CTA images had a limited quality, lacked some information, and could not render a perfect 3D geometry of cerebral arteries with an aneurysm. A minor part of the geometry was assumed. Small blood vessels were not considered in the 3D geometry of the cerebral arteries with an aneurysm. The effect of the entire flow was assumed to be small and negligible. Due to the lack of experimental studies involving a twophase blood model, experimental data that were obtained within the laminar flow region were selected. Moreover, only three patientspecific aneurysm geometries were selected for the numerical simulation of the two-phase blood flow model, but these three geometries may not generally represent the entire population of cerebral artery aneurysms. The two phases of blood, namely, plasma and RBCs, were considered in this study; the WBCs were not considered and were assumed as not having a significant impact on the flow given that their volume percentage is $1 \%$ compared with that of RBCs, which is typically $45 \%$. In the current DPM simulation, the RBCs were assumed to be rigid spherical particles. RBC agglomerations were neglected and were considered as simply spherical particles. The number of particles injected ranged from 145,865 to 185,715 , which is possibly lower than the actual number of RBCs in the blood. The twophase blood flow was solved in a continuous phase using the Navier- 
Stokes equation, and the particulate phase was solved with the DPM using the Lagrangian frame approach. The discrete phase trajectory was calculated using the formulation that included the discrete phase inertia and drag force. The zero-pressure outlet for the boundary conditions was chosen due to the limitation in obtaining the actual pressure data. The vessel wall was considered rigid with a no-slip boundary condition. Moreover, rupture risk was predicted based on published values. A high risk of rupture was associated with high WSS, high OSI, and long RRT. In the analysis of rupture risk, low WSS was indicated by $<1 \mathrm{~Pa}$, whereas high WSS was indicated by $>3 \mathrm{~Pa}$ in the brain vessel. OSI indicated the directional changes in WSS, and it ranged between 0 and 0.2 , where 0 indicated no oscillation and 0.2 indicated a high oscillation. RRT was either long or short based on the contour value. A contour value between 8 and 14 indicated a long RRT, whereas a value of 7 or less indicated a short RRT.

\subsection{CONCLUSION}

The blood flow in the three aneurysm geometries was numerically simulated using the DPM, which considered the two-phase nature of blood. Three important hemodynamic parameters, namely, WSS, OSI, and RRT, were analyzed. The simulation of the two-phase blood flow revealed a high WSS $(>3.0 \mathrm{~Pa})$, a high OSI $(>0.2)$, and a long RRT $(>8)$ in medium- and giant-sized saccular aneurysms. In the investigated region, high stress, high flow oscillation, and flow recirculation may be associated with a high risk of aneurysm rupture. For a medium-sized fusiform aneurysm located in the CCA, a low OSI, a low WSS, and a short RRT were found at the aneurysm dome. The risk of rupture in the bulge area may be lower compare to other area. However, at the bifurcation point, a high WSS and a long RRT with low oscillation were observed. The aneurysm may tend to grow at the bifurcation area where velocity is high. The results suggested the need to comprehensively analyze the correlation between hemodynamic parameters and the risk of rupture. Given the limitations highlighted in this study, in-depth and detailed numerical simulation of a larger number of patient-specific geometries is warranted. This paper underscores the importance of biomechanical forces in the risk of aneurysm rupture.

\subsection{DECLARATION OF COMPETING INTEREST}

The authors declare that they have no known competing financial interests or personal relationships that could have appeared to influence the work reported in this paper.

\subsection{ACKNOWLEDGMENTS}

The authors acknowledge the financial support provided by Taylor's 
University Malaysia through Taylor's University Flagship Research Programme (TUFR) and Taylor's Research Excellence Scholarship.

\subsection{REFERENCES}

[1] N. Chalouhi, B. L. Hoh, and D. Hasan, "Review of cerebral aneurysm formation, growth, and rupture," Stroke, vol. 44, no. 12, pp. 3613-3622, 2013.

[2] J. Menke, J. Larsen, and K. Kallenberg, "Diagnosing cerebral aneurysms by computed tomographic angiography: meta-analysis," Ann. Neurol., vol. 69, no. 4, pp. 646-654, 2011.

[3] A. I. Shehata, T. Hassan, and K. M. Saqr, "Effects of Non-Newtonian Viscosity on the Hemodynamics of Cerebral Aneurysms," Appl. Mech. Mater., vol. 819, pp. 366-370, 2016.

[4] M. Longo et al., "Role of Hemodynamic Forces in Unruptured Intracranial Aneurysms: An Overview of a Complex Scenario," World Neurosurg., vol. 105, pp. 632-642, 2017.

[5] S. Omodaka et al., "Local hemodynamics at the rupture point of cerebral aneurysms determined by computational fluid dynamics analysis," Cerebrovasc. Dis., vol. 34, no. 2, pp. 121-129, 2012.

[6] A. Mantha, C. Karmonik, G. Benndorf, C. Strother, and R. Metcalfe, "Hemodynamics in a cerebral artery before and after the formation of an aneurysm," Am. J. Neuroradiol., vol. 27, no. 5, pp. 1113-1118, 2006.

[7] S. Sugiyama et al., "Computational hemodynamic analysis for the diagnosis of atherosclerotic changes in intracranial aneurysms: a proofof-concept study using 3 cases harboring atherosclerotic and nonatherosclerotic aneurysms simultaneously," Comput. Math. Methods Med., vol. 2016, 2016.

[8] N. Varble, K. Kono, H. Rajabzadeh-Oghaz, and H. Meng, "Rupture Resemblance Models May Correlate to Growth Rates of Intracranial Aneurysms: Preliminary Results," World Neurosurg., vol. 110, pp. e794e805, 2018.

[9] P. M. Munarriz, P. A. Gómez, I. Paredes, A. M. Castaño-Leon, S. Cepeda, and A. Lagares, "Basic Principles of Hemodynamics and Cerebral Aneurysms," World Neurosurg., vol. 88, pp. 311-319, 2016.

[10] Y. Wang, X. Leng, X. Zhou, W. Li, A. H. Siddiqui, and J. Xiang, "Hemodynamics in a middle cerebral artery aneurysm before its growth and fatal rupture: Case study and review of the literature," World Neurosurg., vol. 119, pp. e395-e402, 2018.

[11] G. Carty, S. Chatpun, and D. M. Espino, "Modeling blood flow through intracranial aneurysms: A comparison of Newtonian and nonNewtonian viscosity," J. Med. Biol. Eng., vol. 36, no. 3, pp. 396-409, 2016.

[12] A. Valencia, H. Morales, R. Rivera, E. Bravo, and M. Galvez, "Blood flow dynamics in patient-specific cerebral aneurysm models: the relationship between wall shear stress and aneurysm area index," Med. Eng. Phys., 
vol. 30, no. 3, pp. 329-340, 2008.

[13] C. Ou, W. Huang, M. M. F. Yuen, and Y. Qian, "Hemodynamic modeling of leukocyte and erythrocyte transport and interactions in intracranial aneurysms by a multiphase approach," J. Biomech., vol. 49, no. 14, pp. 3476-3484, 2016.

[14] M. Dhahbi, M. Ben Chiekh, B. Gilles, J. C. Béra, and A. Jemni, "Numerical simulations of particle dynamics in a poststenotic blood vessel region within the scope of extracorporeal ultrasound stenosis treatment," Med. Eng. Phys., vol. 34, no. 7, pp. 982-989, 2012.

[15] S. A. J. Morsi and A. J. Alexander, "An investigation of particle trajectories in two-phase flow systems," J. Fluid Mech., vol. 55, no. 2, pp. 193-208, 1972.

[16] I. ANSYS, "Fluent 6.2 User's Manual." ANSYS, Inc. Canonsburg, PA, 2005.

[17] T. Karino and H. L. Goldsmith, "Flow behaviour of blood cells and rigid spheres in an annular vortex," Philos. Trans. R. Soc. London. B, Biol. Sci., vol. 279, no. 967, pp. 413-445, 1977.

[18] J. Jung and A. Hassanein, "Three-phase CFD analytical modeling of blood flow," Med. Eng. Phys., vol. 30, no. 1, pp. 91-103, 2008.

[19] K. Joisar, R. Bhoraniya, and A. Harichandan, "Numerical Analysis of Two-Phase Blood Flow in Idealized Artery with Blockage," in Innovative Design, Analysis and Development Practices in Aerospace and Automotive Engineering (I-DAD 2018), Springer, 2019, pp. 259-267.

[20] S. K. Yu and H. L. Goldsmith, "Behavior of model particles and blood cells at spherical obstructions in tube flow," Microvasc. Res., vol. 6, no. 1, pp. 5-31, 1973.

[21] P. A. Thrower, "global research conference (grace 2020)," 2020. .

[22] K. Valen-Sendstad, K.-A. Mardal, M. Mortensen, B. A. P. Reif, and H. P. Langtangen, "Direct numerical simulation of transitional flow in a patient-specific intracranial aneurysm," J. Biomech., vol. 44, no. 16, pp. 2826-2832, 2011.

[23] H. Meng, V. M. Tutino, J. Xiang, and A. Siddiqui, "High WSS or low WSS? Complex interactions of hemodynamics with intracranial aneurysm initiation, growth, and rupture: toward a unifying hypothesis," Am. J. Neuroradiol., vol. 35, no. 7, pp. 1254-1262, 2014.

[24] N. Varble et al., "Shared and distinct rupture discriminants of small and large intracranial aneurysms," Stroke, vol. 49, no. 4, pp. 856-864, 2018.

[25] N. Varble, K. Kono, H. Rajabzadeh-Oghaz, and H. Meng, "Rupture resemblance models may correlate to growth rates of intracranial aneurysms: preliminary results," World Neurosurg., vol. 110, pp. e794e805, 2018.

[26] J. Xiang et al., "Hemodynamic-morphologic discriminants for intracranial aneurysm rupture," Stroke, vol. 42, no. 1, pp. 144-152, 2011.

[27] S. Soldozy et al., "The biophysical role of hemodynamics in the pathogenesis of cerebral aneurysm formation and rupture," Neurosurg. Focus, vol. 47, no. 1, p. E11, 2019. 
[28] M. A. A. Sheikh, A. S. Shuib, and M. H. H. Mohyi, "A Review of Hemodynamic Parameters in Cerebral Aneurysm," Interdiscip. Neurosurg., p. 100716, 2020.

[29] P. Jiang et al., "Hemodynamic characteristics associated with thinner regions of intracranial aneurysm wall," J. Clin. Neurosci., vol. 67, pp. 185190, 2019.

[30] P. R. Hoskins and D. Hardman, "Three-dimensional imaging and computational modelling for estimation of wall stresses in arteries," $\mathrm{Br}$. J. Radiol., vol. 82, no. special_issue_1, pp. S3-S17, 2009.

[31] J. M. Dolan, J. Kolega, and H. Meng, "High wall shear stress and spatial gradients in vascular pathology: a review," Ann. Biomed. Eng., vol. 41, no. 7, pp. 1411-1427, 2013.

[32] D. M. Sforza, C. M. Putman, and J. R. Cebral, "Hemodynamics of cerebral aneurysms," Annu. Rev. Fluid Mech., vol. 41, pp. 91-107, 2009.

[33] T. Qiu, G. Jin, H. Xing, and H. Lu, "Association between hemodynamics, morphology, and rupture risk of intracranial aneurysms: a computational fluid modeling study," Neurol. Sci., vol. 38, no. 6, pp. 1009-1018, 2017.

[34] S. Sugiyama et al., "Relative residence time prolongation in intracranial aneurysms: a possible association with atherosclerosis," Neurosurgery, vol. 73, no. 5, pp. 767-776, 2013. 